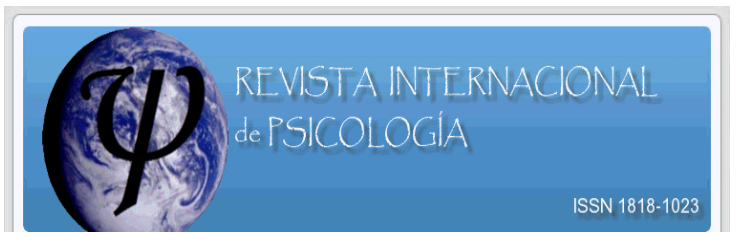

\title{
ESTRÉS DE EXAMEN, VARIABLES MODULADORAS Y REPROBACIÓN ESCOLAR
}

Dr. Arturo Barraza Macías, Ma. Oscar Rodríguez ${ }^{1}$

\begin{abstract}
RESUMEN
La presente investigación se planteó cuatro objetivos: a) establecer el perfil descriptivo del estrés de examen de los alumnos de educación media superior, b)identificar las materias que más estrés provocan en los alumnos de educación media superior al momento de presentar un examen, c) distinguir las variables sociodemográficas y situacionales que establecen diferencias significativas en el estrés de examen de los alumnos de educación media superior y d) determinar la relación que existe entre el estrés de examen y el número de materias reprobadas en los alumnos de educación media superior. Se fundamenta teóricamente en el modelo sistémico cognoscitivista del estrés académico que se deriva de la vertiente transaccionalista del Programa de Investigación Persona-Entorno. Para el logro de dichos objetivos se realizó un estudio transeccional, no experimental y correlacional mediante la aplicación del Inventario del Estrés de Examen a 343 alumnos del Colegio de Bachilleres Lomas de la ciudad de Durango, en el estado de Durango, México. Sus principales resultados permiten establecer un perfil descriptivo del estrés de examen de los alumnos de educación media superior, así como confirmar el papel modulador de las variables género y semestre y la correlación positiva entre el estrés de examen y el número de materia reprobadas.
\end{abstract}

\section{PALABRAS CLAVES}

estrés de examen, estresores, síntomas y estrategias de afrontamiento

\begin{abstract}
This research was raised four objectives: a) to establish the profile of stress descriptive review of the higher education students, b) identify areas that cause more stress on the students of higher education when presenting a review c ) distinguish sociodemographic variables and situational which provide significant differences in stress examination of students in higher education d) determining the relationship between stress examination and the number of subjects disapproved of the students in higher education. It is based on the model theoretically systemic cognoscitivista academic stress which is derived from the slope of transaccionalista Research Program Person-environment. To achieve these goals, a study was conducted transectional not experimental and correlational through the implementation of the Inventory Stress Test to 343 students, of the Preparatory Lomas of the city of Durango, in the state of Durango, Mexico. Its main results allow establishing a descriptive profile of stress examination of students in higher education, as well as confirm the modulatory effect of the variables and gender semester and the positive correlation between stress and the number of examination subject disapproved.
\end{abstract}

\footnotetext{
${ }^{1}$ Universidad Pedagógica de Durango. Correos electrónicos: tbarraza@terra.com.mx barraza_upd@terra.com.mx
} 


\section{KEY WORD \\ Stress Test, Sources of stress, Symptoms and Coping strategies}

\section{INTRODUCCIÓN}

Todas las personas suelen presentar estrés al enfrentarse a situaciones en las que se juegan algo importante. En el ámbito académico, una de las principales situaciones donde el alumno se juega algo importante, o quizás lo más importante, (su acreditación y, por ende, su permanencia en la institución) es el examen; en ese sentido, es que suele considerase como una situación normal que el alumno al enfrentarse a un examen manifieste estrés.

Una de las primeras investigaciones que abordaron el estrés que se genera a partir de un examen es la realizada por Mechanic en 1962 (en Lazarus y Folkman, 1986), quien estudió la experiencia de un grupo de estudiantes en torno a la preparación y realización de sus exámenes de doctorado. A partir de esa fecha, y bajo la misma lógica, se han realizado múltiples investigaciones que abordan el estrés a partir de una situación generada por un examen (v. gr. Pellicer, Salvador y Benet, 2002; Salgero, 1989; Viñas y Caparrós, 2000).

Estas investigaciones parten del hecho de reconocer al examen como una situación generadora de estrés; sin embargo, ninguna ha estudiado al examen como una situación estresante en sí misma. Esta circunstancia condujo a Barraza y Acosta (2007) a abordar el estudio del estrés de examen y considerarlo un tipo de estrés derivado del estrés académico. A partir de ese trabajo se abre una línea de investigación que tiene por objeto de estudio al estrés de examen.

\section{Planteamiento del problema}

El asistir diario a la escuela, el hacer las tareas y trabajos escolares, el participar en clase y el ponerle atención al maestro no bastan para acreditar una materia en la gran mayoría de nuestras escuelas de educación media superior. Ineludiblemente el estudiante se tiene que enfrentar a un período de exámenes que, más allá de su carácter obligatorio, nunca es bien recibido por los estudiantes.

No importa el tiempo que se lleve estudiando, los nervios siempre aparecen en mayor o menor medida. Esta situación se considera algo normal entre los profesores, y entre los estudiantes, y en la mayoría de los casos se piensa que el estar nervioso, estresado y con cierta dosis de ansiedad puede ayudar a ponerle manos a la obra y a mantener un rendimiento alto. Más allá de estas creencias, la realidad es que en algunos alumnos el estrés puede llegar a ser muy intenso, a tal grado que interfiere en su concentración y rendimiento.

Nuestra postura al respecto, es que más allá de creencias arquetípicas se hace necesario constituir al estrés de examen en un objeto de investigación susceptible de indagación empírica; en ese sentido es que se plantea la presente investigación, la cual pretende lograr los siguientes objetivos:

Establecer el perfil descriptivo del estrés de examen de los alumnos de educación media superior. 
Identificar las materias que más estrés provocan en los alumnos de educación media superior al momento de presentar un examen.

Distinguir las variables sociodemográficas y situacionales que establecen diferencias significativas en el estrés de examen de los alumnos de educación media superior.

Determinar la relación que existe entre el estrés de examen y el número de materias reprobadas en los alumnos de educación media superior.

\section{Antecedentes}

La presente investigación tiene como antecedente general el estado del arte sobre el estrés académico elaborado por Barraza (2007a) y como antecedentes específicos tres investigaciones: Barraza (2005), Barraza y Acosta (2007) y Viñas y Caparrós (2000).

En lo que corresponde al estado del arte sobre el estrés académico se puede observar que éste reporta la existencia de los siguientes estresores:

Falta de tiempo o tiempo limitado para poder cumplir con las actividades académicas.

Sobrecarga académica, de estudio, de tareas o de trabajo escolares.

Realización de un examen.

Exposición de trabajos en clase.

Realización de trabajos obligatorios para aprobar la asignatura.

Exceso de responsabilidad por cumplir las obligaciones escolares.

La tarea de estudio.

El tipo de trabajo que le piden los profesores. .

Intervención en el aula.

Mantener un buen rendimiento o promedio académico.

La evaluación de los profesores.

Como se puede advertir, entre los estresores reportados por la literatura consultada para la elaboración del estado del arte se encuentra la realización de un examen, lo que conduce a reconocer al examen como fuente generadora de estrés.

En lo específico, los antecedentes para esta investigación se pueden encontrar en tres investigaciones:

a) En la investigación desarrollada por Barraza (2005) se abordó el estudio del estrés académico en los alumnos de educación media superior. El cuestionario fue aplicado a 356 estudiantes de tres instituciones y entre sus principales resultados se presentan los siguientes:

El 86\% de los estudiantes declaran haber tenido estrés académico, con nivel medianamente alto, durante el semestre enero-junio 2004.

Los estudiantes atribuyen este estrés al exceso de responsabilidad por cumplir las obligaciones escolares, sobrecarga de tareas y trabajos escolares, evaluación de los profesores y el tipo de trabajo solicitado por los docentes. Los síntomas que se presentan con mayor intensidad son los psicológicos. 
b) La investigación desarrollada por Barraza y Acosta (2007) abordó el estudio del estrés de examen en una institución de educación media superior. El cuestionario se aplicó a 351 estudiantes y entre sus principales resultados se encuentran los siguientes:

El $81 \%$ de estudiantes reportan haber presentado estrés durante el periodo de exámenes

Las demandas del entorno que son consideradas estresores, con mayor nivel de frecuencia son: la calificación que pudiera obtenerse y el tipo de preguntas, ejercicios o problemas que se encuentran en el examen.

Se observó el predominio absoluto de los síntomas psicológicos, entre los diferentes tipos de síntomas,

Las estrategias de afrontamiento utilizadas con mayor frecuencia fueron: volver a repasar los apuntes o ejercicios para el examen y platicar con los compañeros

El análisis de diferencia de grupos permitió reconocer que las variables género y semestre presentan un papel modulador en este tipo de estrés.

c) La investigación desarrollada por Viñas y Caparrós (2000), tuvo como principal objetivo analizar la posible relación entre las estrategias utilizadas por 120 estudiantes universitarios para afrontar el periodo de exámenes y los síntomas somáticos autoinformados. Para ello, evaluaron las estrategias de afrontamiento utilizadas durante las dos semanas correspondientes a la primera convocatoria de exámenes. De forma paralela, los estudiantes autoinformaron de los diferentes tipos de quejas y manifestaciones somáticas experimentadas durante dicho periodo y a lo largo de las dos semanas consecutivas.

Los resultados obtenidos coinciden con los de otros estudios, en los cuales se observa que las estrategias de afrontamiento focalizadas en el problema (afrontamiento activo de la situación) están relacionadas con un mayor bienestar físico. Por el contrario, aquellos sujetos que utilizan estrategias paliativas, como el afrontamiento centrado en las emociones, el escape conductual o cognitivo, expresan mayor malestar.

\section{MARCO TEÓRICO}

\section{Ubicación teórica del modelo utilizado}

El estudio del estrés académico, en lo general, o del estrés de examen, en lo particular, se puede realizar desde tres perspectivas que definen tres esfuerzos de sistematización y organización teórica:

La perspectiva del discurso único, al cual se denomina Enfoque Teórico del Estrés (Benjamín, 1992).

La perspectiva de los enfoques conceptuales, que define para el estudio del estrés tres enfoques diferentes: el centrado en los estresores, el centrado en los síntomas y el centrado en la interacción del sujeto con su entorno (Elliot y Eisdorfer, 1982; Travers y Cooper, 1997; etc.).

La perspectiva de los Programas de Investigación rivales, que identifica dos programas de investigación que guían la indagación empírica del campo de estudio del estrés: el Programa de Investigación Estímulo-Respuesta y el Programa de Investigación Persona-Entorno (Barraza, 2007b) 
En la presente investigación nos adscribimos a la tercera perspectiva y asumimos el Programa de Investigación Persona-Entorno. El núcleo teórico base de este Programa de Investigación se constituye, entre otros, por los siguientes postulados:

Postulado Base: el estrés es conceptualizado como la interacción dinámica que se establece entre la persona y su entorno.

Postulado Uno: el estrés es la representación interna de transacciones particulares y problemáticas entre la persona y su entorno.

Postulado Dos: la valoración de las demandas del entorno y de los recursos de las personas para enfrentar esas demandas se desarrolla en dos momentos, en el primero se identifica el problema y en el segundo la forma de enfrentarlo.

Los modelos desarrollados bajo el Programa de Investigación Persona-Entorno pueden agruparse en dos grandes variantes: la interaccional y la transaccional, dentro de los primeros, destaca por méritos propios el modelo de demanda-control (Karasek, 1979; Karasek, 2001; y Theorell y Karasek, 1996), mientras que en el caso de los segundos el panorama es dominado por el modelo transaccional (Cohen y Lazarus, 1979; Lazarus, 2000; y Lazarus y Folkman, 1986). La atención en el presente trabajo esta centrada en la perspectiva transaccionalista.

Con relación al modelo transaccional se puede afirmar que éste se centra básicamente en los procesos cognitivos que se desarrollan en torno a una situación estresante que es el resultado de las transacciones entre la persona y el entorno. Estas transacciones dependen del impacto del estresor ambiental, impacto que es mediatizado en primer lugar por las evaluaciones que hace la persona del estresor y, en segundo lugar, por los recursos personales, sociales o culturales disponibles para hacer frente a la situación de estrés. Con base en este modelo y en la Teoría General de Sistemas (Bertalanfy, 1991) se elaboró el modelo sistémico cognoscitivista del estrés académico (Barraza, 2006), el cual constituye el primer modelo elaborado de manera específica para el estrés académico y que en la presente investigación se toma como fundamento teórico para el estudio del estrés de examen.

\section{El modelo sistémico cognoscitivista del estrés académico}

Las hipótesis constitutivas del modelo sistémico cognoscitivista del estrés académico son las siguientes:

Hipótesis de los componentes sistémicos-procesuales del estrés académico.

Hipótesis del estrés académico como estado psicológico

Hipótesis de los indicadores del desequilibrio sistémico que implica el estrés académico

La hipótesis del afrontamiento como restaurador del equilibrio sistémico

Estas hipótesis permiten una lectura sistémico-cognoscitivista del estrés académico, y del estrés de examen, en los siguientes términos (vid cuadro infra): 1.- El entorno le plantea a la persona un conjunto de demandas o exigencias, en este caso de evaluación.

2.- Estas demandas son sometidas a un proceso de valoración por parte de la persona,

3.- En el caso de considerar que las demandas desbordan sus recursos las valora como estresores.

4.- Estos estresores se constituyen en el input que entra al sistema y provoca un desequilibrio sistémico en la relación de la persona con su entorno. 
5.- El desequilibrio sistémico da paso a un segundo proceso de valoración de la capacidad de afrontar (coping) la situación estresante, lo que determina cuál es la mejor manera de enfrentar esa situación.

6.- El sistema responde con estrategias de afrontamiento (output) a las demandas del entorno.

7.- Una vez aplicadas estas estrategias de afrontamiento, en caso de ser exitosas, el sistema recupera su equilibrio sistémico; en caso de que las estrategias de afrontamiento no sean exitosas el sistema realiza un tercer proceso de valoración que lo conduce a un ajuste de las estrategias para lograr el éxito esperado.

Ilustración No. 1 El modelos sistémico cognoscitivista del estrés académico

\section{MODELO SISTÉMICO COGNOSCITIVISTA DEL ESTRÉS ACADÉMICO}

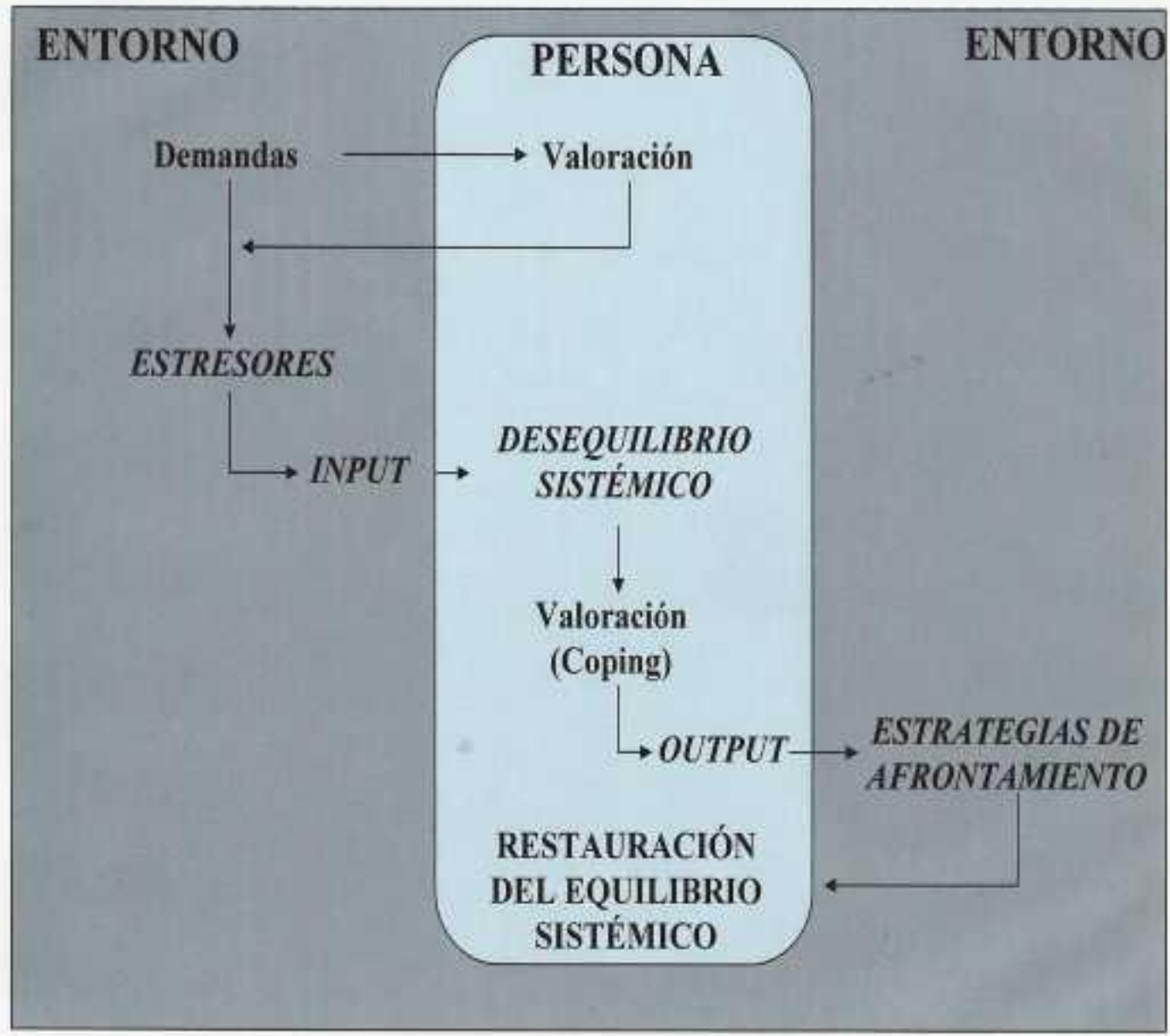

\section{Conceptualización del estrés de examen}

En consonancia con el modelo presentado, se elabora el presente constructo teórico del estrés de examen, el cual intenta recuperar su carácter procesual y su constitución multidimensional: el estrés de examen es un proceso sistémico, de carácter adaptativo y esencialmente psicológico, que se presenta de manera descriptiva en tres momentos: 
Primero: el alumno se ve sometido, en contextos escolares, a una serie de demandas de evaluación que, bajo la valoración del propio alumno son consideradas estresores (input), Segundo: esos estresores provocan un desequilibrio sistémico (situación estresante) que se manifiesta en una serie de síntomas (indicadores del desequilibrio)

Tercero: ese desequilibrio sistémico obliga al alumno a realizar acciones de afrontamiento (output) para restaurar el equilibrio sistémico.

\section{METODOLOGÍA}

Con base en las diferentes perspectivas que se pueden utilizar para clasificar un estudio (Namakforoosh, 2002), este estudio se puede caracterizar como: a) transeccional, por su dimensión temporal, ya que este tipo de estudios recolectan la información una sola vez, b) no experimental, por el control de las variables, ya que este tipo de estudios se realizan sin controlar las variables y reportan solo lo que sucede, y c) correlacional, por la naturaleza de la relación entre variables, ya que este tipo de estudios "tienen como propósito medir el grado de relación que existe entre dos o más conceptos o variables (en un contexto particular)" (Hernández, Fernández y Baptista, 1998; p. 62).

Para la recolección de información se utilizó como técnica la encuesta y como instrumento el Inventario del Estrés de Examen; este inventario se encuentra conformado por 29 items distribuidos de la siguiente manera:

Un ítem de filtro que, en términos dicotómicos (si-no), permite determinar si el encuestado es candidato o no a contestar el inventario.

Un ítem que, en un escalamiento tipo Lickert de cinco valores numéricos (del 1 al 5, donde uno es poco y cinco mucho), permite identificar el nivel de intensidad del estrés de examen.

Siete ítems que, en un escalamiento tipo Lickert de cinco valores categoriales (nunca, rara vez, algunas veces, casi siempre y siempre), permiten identificar la frecuencia en que las demandas de evaluación del entorno son valoradas como estímulos estresores.

14 ítems que, en un escalamiento tipo Lickert de cinco valores categoriales (nunca, rara vez, algunas veces, casi siempre y siempre), permiten identificar la frecuencia con que se presentan los síntomas o reacciones al estímulo estresor.

Seis ítems que, en un escalamiento tipo lickert de cinco valores categoriales (nunca, rara vez, algunas veces, casi siempre y siempre), permiten identificar la frecuencia de uso de las estrategias de afrontamientos.

Este inventario presenta las siguientes propiedades psicométricas:

a) Una confiabilidad en alfa de Cronbach de .88. Este nivel de confiabilidad puede ser valorado como elevado, según Murphy y Davishofer (en Hogan, 2004).

b) Una estructura tridimensional que se confirma a través de la estructura factorial obtenida en el análisis correspondiente, lo cual coincide con el modelo conceptual sistémico cognoscitivista adoptado para el estudio del estrés de examen.

c) Una homogeneidad y direccionalidad única de los ítems, establecida a través del análisis de consistencia interna y de grupos contrastados. Estos resultados centrados 
en la relación entre los ítems y el puntaje global del inventario permiten afirmar que todos los ítems forman parte del constructo establecido en el modelo conceptual construido, en este caso, el estrés de examen.

La población de estudio se conformó por 2250 alumnos que asisten a clases de manera regular en el Colegio de Bachilleres Lomas de la ciudad de Durango, en el estado de Durango. México, en sus dos turnos. El tamaño de la muestra se obtuvo mediante el programa estadístico STATS versión dos a partir de los siguientes valores:

Error máximo aceptable 5\%

Porcentaje estimado de la muestra 50\%

Nivel deseado de confianza $95 \%$

La muestra quedó constituida por 328 alumnos; para la selección de los alumnos a encuestar se siguió un muestreo de tipo probabilístico con un procedimiento por conglomerado, que condujo a identificar a los grupos de alumnos como unidades muestrales. El cuestionario se aplicó los días 28, 29 y 30 de abril de 2007. En total se recolectaron 343 cuestionarios y la distribución de los encuestados según las variables sociodemográficas estudiadas fue la siguiente:

$51 \%$ correspondió al género masculino y $49 \%$ restante al género femenino. $12.2 \%$ tenían 15 años de edad; 27.4\% 16 años; $31.8 \% 17$ años; $22.2 \% 18$ años, y el $6.4 \% 19$ años.

$30 \%$ terminó de cursar el primer semestre; $31.8 \%$ el tercer semestre y $38.2 \%$ el quinto semestre.

$50.7 \%$ asistían a clases en el turno matutino y el $49.3 \%$ en el turno vespertino.

\section{RESULTADOS}

Los resultados obtenidos se presentan a partir de los tres momentos de análisis realizados: análisis descriptivo, análisis de diferencia de grupos y análisis correlacional.

\section{Análisis descriptivo}

a) Estresores

La media obtenida en cada uno de los estresores que indaga el Inventario se presenta en la siguiente tabla:

Tabla No. 1 Media obtenida en cada uno de los estresores

\begin{tabular}{|l|c|}
\hline \multicolumn{1}{|c|}{ ESTRESORES } & Media \\
\hline El no haber estudiado previamente & 3,09 \\
\hline La calificación que pudieras obtener & $\mathbf{3 , 9 0}$ \\
\hline El tipo de preguntas, ejercicios, o problemas que se encuentran en el examen & $\mathbf{3 , 4 6}$ \\
\hline La falta de tiempo para terminar el examen & 2,94 \\
\hline La forma en que el profesor va a calificar el examen & 3,22 \\
\hline Olvidar lo que estudiaste en el momento del examen & 3,17 \\
\hline La vigilancia que hiciera el profesor para que no pudieras copiar & 2,60 \\
\hline
\end{tabular}

Como se puede observar, las demandas del entorno que son valoradas con mayor frecuencia como estresores por los alumnos del Colegio de Bachilleres Lomas son la calificación que pudiera obtener y el tipo de preguntas, ejercicios, o problemas que se encuentran en el examen, mientras que las demandas del entorno que son valoradas 
con menor frecuencia como estresores por los alumnos del Colegio de Bachilleres Lomas son la vigilancia que hiciera el profesor para que no pudiera copiar y la falta de tiempo para terminar el examen.

\section{b) Síntomas}

La media obtenida en cada uno de los síntomas que indaga el Inventario se presenta en la siguiente tabla:

Tabla No. 2 Medias obtenidas en cada uno de los síntomas

\begin{tabular}{|l|c|}
\hline \multicolumn{1}{|c|}{ SÍNTOMAS } & Media \\
\hline Trastornos en el sueño (insomnio o pesadillas) & 1,89 \\
\hline Fatiga crónica (cansancio permanente) & 2,12 \\
\hline Problemas de digestión, dolor abdominal o diarrea & 1,46 \\
\hline Rascarse, morderse las uñas, frotarse, etc. & $\mathbf{2 , 6 2}$ \\
\hline Somnoliencia o mayor necesidad de dormir & 2,10 \\
\hline Sentimientos de depresión y tristeza (decaído) & 2,35 \\
\hline Problemas de concentración & $\mathbf{3 , 0 4}$ \\
\hline Sensación de tener la mente vacía (bloqueo mental) & 2,54 \\
\hline Sentimiento de agresividad o aumento de irritabilidad & 2,16 \\
\hline Conflictos o discusiones con mis compañeros & 1,78 \\
\hline Aislamiento de los demás & 1,80 \\
\hline Desgano para realizar las labores escolares & 2,40 \\
\hline Absentismo e impuntualidad en las clases & 1,98 \\
\hline Aumento o reducción del consumo de alimentos & 2,11 \\
\hline
\end{tabular}

Como se puede observar los síntomas que se presentan con mayor frecuencia entre los alumnos del Colegio de Bachilleres Lomas son problemas de concentración y rascarse, morderse las uñas, frotarse, etc., mientras que los que se presentan con menor frecuencia son problemas de Digestión, dolor abdominal o diarrea y conflictos o discusiones con mis compañeros.

c) Estrategias de afrontamiento

La media obtenida en cada uno de las estrategias de afrontamiento que indaga el Inventario se presenta en la siguiente tabla:

Tabla No. 3 Medias obtenidas en cada una de las estrategias de afrontamiento

\begin{tabular}{|l|c|}
\hline \multicolumn{1}{|c|}{ ESTRATEGIAS DE AFRONTAMIENTO } & Media \\
\hline Volver a repasar los apuntes o ejercicios para el examen & $\mathbf{3 , 3 4}$ \\
\hline Esforzarse por mantener la calma (respiración o concentración profunda) & 2,97 \\
\hline Elogios a sí mismo (echarme porras y convencerme de que si puedo) & 2,91 \\
\hline Distracción evasiva (salir a caminar, comprar, saludar a alguien, etc.) & 2,47 \\
\hline La religiosidad (realizar oraciones) & 2,14 \\
\hline Platicar con los compañeros (hablar sobre el examen, la materia, o el maestro) & $\mathbf{3 , 4 0}$ \\
\hline
\end{tabular}

Como se puede observar las estrategias de afrontamiento que son utilizadas con mayor frecuencia por los alumnos del Colegio de Bachilleres Lomas son platicar con los compañeros (hablar sobre el examen, la materia, o el maestro) y volver a repasar los apuntes o ejercicios para el examen, mientras que las utilizada con menor frecuencia son la religiosidad (realizar oraciones) y Distracción evasiva (salir a caminar, comprar, saludar a alguien, etc.).

d) Estrés de examen

El porcentaje obtenido en cada uno de los aspectos indagados sobre el estrés de examen fueron los siguientes: 
Tabla No. 4 Porcentaje obtenido en cada uno de los aspectos indagados del estrés de examen

\begin{tabular}{|l|c|}
\hline \multicolumn{1}{|c|}{ ASPECTOS } & PORCENTAJE \\
\hline Presencia & $96.8 \%$ \\
\hline Frecuencia & $50 \%$ \\
\hline Intensidad & $64 \%$ \\
\hline
\end{tabular}

Estos resultados, interpretados con el baremo utilizado (vid infra) permiten afirmar que el 96.8\% de los alumnos del Colegio de Bachilleres Lomas algunas veces presentan el estrés de examen con una intensidad medianamente alta.

Tabla No. 5 Baremos utilizados para la interpretación de la frecuencia e intensidad del estrés de examen

\section{Baremo Intensidad}

\begin{tabular}{|l|c|}
\hline Rango & Categoría \\
\hline O a 20 & Bajo \\
\hline 21 a 40 & Medianamente bajo \\
\hline 41 a 60 & Medio \\
\hline 61 a 80 & Medianamente alto \\
\hline 81 a 100 & Alto \\
\hline
\end{tabular}

\section{Baremo Frecuencia}

\begin{tabular}{|l|c|}
\hline Rango & Categoría \\
\hline O a 20 & Nunca \\
\hline 21 a 40 & Rara vez \\
\hline 41 a 60 & Algunas veces \\
\hline 61 a 80 & Casi siempre \\
\hline 81 a 100 & Siempre \\
\hline
\end{tabular}

e) Materias que provocan estrés de examen.

Los resultados obtenidos en la pregunta sobre que materias le preocupaban más a la hora de presentar un examen, se presentan en la siguiente tabla:

Tabla No. 6 Porcentaje que muestra que tanto les estresa cada materia al momento de presentar un examen

\begin{tabular}{|l|l|c|c|c|}
\hline & & Frecuencia & Porcentaje & $\begin{array}{c}\text { Porcentaje } \\
\text { Válido }\end{array}$ \\
\hline Válidos & Ninguna & 1 &, 3 &, 3 \\
\hline & Matemáticas & 103 & 30,0 & 31,0 \\
\hline & Cálculo & 75 & 21,9 & 22,6 \\
\hline & Física & 38 & 11,1 & 11,4 \\
\hline & Filosofía & 37 & 10,8 & 11,1 \\
\hline & Química & 23 & 6,7 & 6,9 \\
\hline & Otra & 55 & 16,0 & 16,6 \\
\hline & Total & 332 & 96,8 & 100,0 \\
\hline Perdidos & $\mathbf{8 8}$ & 11 & 3,2 & \\
\hline Total & & 343 & 100,0 & \\
\hline
\end{tabular}

Como se puede observar, la materia que más estresa a los alumnos a la hora de presentar un examen es Matemáticas, seguida por Cálculo que es una materia con una carga esencialmente matemática; en suma, la matemática preocupa al más del $50 \%$ de los alumnos. 
Con base en los resultados obtenidos en los cinco rubros del análisis descriptivo es posible establecer el siguiente perfil descriptivo del estrés de examen de los alumnos del Colegio de Bachilleres Lomas:

El 96.8\% de los alumnos del Colegio de Bachilleres Lomas algunas veces presentan el estrés de examen con una intensidad medianamente alta.

Con relación a la presencia del estrés de examen se difiere con el reportado por Barraza y Acosta (2007), ya que estos autores reportan un 81\%. Este porcentaje indica una diferencia de casi 16 puntos porcentuales que permite afirmar que un mayor porcentaje de los alumnos del Colegio de Bachilleres Lomas presentan estrés de examen en comparación de los alumnos del Colegio de Ciencias y Humanidades.

Con relación a la frecuencia se observa una diferencia de escasos dos puntos porcentuales entre ambos estudios por lo que se considera que los alumnos del Colegio de Bachilleres Lomas y del Colegio de Ciencias y Humanidades presentan el estrés de examen con la misma frecuencia.

En lo referente a la intensidad se observa una diferencia de seis puntos porcentuales en relación a lo reportado por Barraza y Acosta (2007), por lo que nuevamente se puede afirmar que los alumnos del Colegio de Bachilleres Lomas presentan con mayor intensidad el estrés de examen en comparación de los alumnos del Colegio de Ciencias y Humanidades.

Las demandas del entorno que son valoradas con mayor frecuencia como estresores por los alumnos del Colegio de Bachilleres Lomas son la calificación que pudiera obtener y el tipo de preguntas, ejercicios, o problemas que se encuentran en el examen, Los síntomas que se presentan con mayor frecuencia entre los alumnos del Colegio de Bachilleres Lomas son problemas de concentración y rascarse, morderse las uñas, frotarse, etc. Las estrategias de afrontamiento que son utilizadas con mayor frecuencia por los alumnos del Colegio de Bachilleres Lomas son platicar con los compañeros (hablar sobre el examen, la materia, o el maestro) y volver a repasar los apuntes o ejercicios para el examen.

Estos resultados coinciden con los reportados por Barraza y Acosta (2007) por lo que se puede afirmar que a partir de estos estudios empieza a visualizarse de manera clara el perfil descriptivo del estrés de examen en alumnos de educación media superior.

Más del 50\% de los alumnos reportan presentar estrés de examen con la materia de matemáticas (o relacionada con las matemáticas) por lo que en este punto se coincide con Campos, Ocampo, Padilla, Corrales y Colindres (2005),

\section{Análisis de diferencia de grupos sin atribución causal}

El análisis de diferencia de grupos sin atribución causal se realizó en tres niveles de análisis: indicadores empíricos, componentes y estrés de examen. En cada caso el análisis se realizó con cuatro variables: dos sociodemográficas (género y edad) y dos situacionales (semestre y turno); en todos los casos se consideró que un nivel de significación menor a .05 representaba que dicha variable si establecía diferencias significativas. Se utilizaron los estadísticos t de Students y ANOVA one way y cuando fue necesario se aplicó la prueba de seguimiento de Scheffe. 
a) Indicadores empíricos del estrés de examen

Los resultados obtenidos del análisis realizado en este nivel con cada una de las variables abordadas se presentan en la siguiente tabla:

Tabla No. 7 Resultados del análisis de diferencia de grupos con relación a los indicadores empíricos del estrés de examen

\begin{tabular}{|c|c|c|c|c|}
\hline INDICADORES & Género & Edad & Semestre & Turno \\
\hline El no haber estudiado previamente &, 737 &, 565 & ,990 &, 103 \\
\hline La calificación que pudieras obtener & 000 & ,308 & ,950 & ,126 \\
\hline $\begin{array}{l}\text { El tipo de preguntas, ejercicios, o problemas que se encuentran en } \\
\text { el examen }\end{array}$ & ,027 & ,425 & ,603 & ,836 \\
\hline La falta de tiempo para terminar el examen & 277 & ,391 & ,209 & ,988 \\
\hline La forma en que el profesor va a calificar el examen & 000 & ,369 &, 516 &, 187 \\
\hline Olvidar lo que estudiaste en el momento del examen & 000 & 207 & 699 & ,856 \\
\hline La vigilancia que hiciera el profesor para que no pudieras copiar &, 551 & ,461 & 087 & ,822 \\
\hline Trastornos en el sueño (insomnio o pesadillas) &, 005 & ,853 & ,511 & ,245 \\
\hline Fatiga crónica (cansancio permanente) & 002 & 390 & 275 & 650 \\
\hline Problemas de digestión, dolor abdominal o diarrea & 002 & ,482 & ,642 & 100 \\
\hline Rascarse, morderse las uñas, frotarse, etc. & 000 & 257 & ,999 & 430 \\
\hline Somnolencia o mayor necesidad de dormir &, 058 & 043 & 001 & ,188 \\
\hline Sentimientos de depresión y tristeza (decaído) & 001 & 965 & 491 & ,599 \\
\hline Problemas de concentración &, 026 & ,406 & ,259 & ,694 \\
\hline Sensación de tener la mente vacía (bloqueo mental) & 220 & ,164 & ,984 & ,316 \\
\hline Sentimiento de agresividad o aumento de irritabilidad & 480 &, 227 & 047 &, 375 \\
\hline Conflictos o discusiones con mis compañeros & ,747 & ,481 &, 102 & ,285 \\
\hline Aislamiento de los demás & ,902 & ,833 & ,721 & ,837 \\
\hline Desgano para realizar las labores escolares & ,355 & ,420 & ,520 & ,630 \\
\hline Absentismo e impuntualidad en las clases & ,497 & ,129 & 181 & 646 \\
\hline Aumento o reducción del consumo de alimentos & 001 & ,056 & 075 & 619 \\
\hline Volver a repasar los apuntes o ejercicios para el examen &, 000 &, 037 &, 000 & 124 \\
\hline $\begin{array}{l}\text { Esforzarse por mantener la calma (respiración o concentración } \\
\text { profunda) }\end{array}$ & ,000 & ,993 & ,034 & ,048 \\
\hline $\begin{array}{l}\text { Elogios a sí mismo (echarme porras y convencerme de que si } \\
\text { puedo) }\end{array}$ & ,002 & ,310 & ,056 & ,830 \\
\hline $\begin{array}{l}\text { Distracción evasiva (salir a caminar, comprar, saludar a alguien, } \\
\text { etc.) }\end{array}$ & ,942 & ,776 & ,217 &, 551 \\
\hline La religiosidad (realizar oraciones) & ,001 & ,057 & ,032 &, 004 \\
\hline $\begin{array}{l}\text { Platicar con los compañeros (hablar sobre el examen, la materia, o } \\
\text { el maestro) }\end{array}$ & ,013 & 015 & 019 & 1,000 \\
\hline
\end{tabular}

Como se puede observar la variable género es la que más establece diferencia significativas entre los indicadores empíricos del estrés de examen (17 de 27), mientras que la variable que menos establece diferencias en el estrés de examen es el turno ( 2 de 27).

b) Componentes del estrés de examen

Los resultados obtenidos del análisis realizado en este nivel con cada una de las variables abordadas se presentan en la siguiente tabla: 
Tabla No. 8 Resultados del análisis de diferencia de grupos con relación a los componentes procesuales del estrés de examen

\begin{tabular}{|l|c|c|c|c|}
\hline \multicolumn{1}{|c|}{ COMPONENTES } & Género & Edad & Semestre & Turno \\
\hline Estresores & $\mathbf{. 0 0 0}$ & .693 & .407 & .710 \\
\hline Síntomas & $\mathbf{. 0 0 0}$ & .318 & .174 & .645 \\
\hline Estrategias de Afrontamiento & $\mathbf{. 0 0 0}$ & .088 & .001 & $\mathbf{. 0 7 2}$ \\
\hline
\end{tabular}

Como se puede observar la variable género establece diferencias significativas en los tres componentes, mientras que la variable semestre en las estrategias de afrontamiento.

c) El estrés de examen

Los resultados obtenidos del análisis realizado en cada uno de los aspectos de este nivel con cada una de las variables abordadas se presentan en la siguiente tabla:

Tabla No. 9 Resultados del análisis de diferencia de grupos con relación a los dos aspectos indagados del estrés de examen

\begin{tabular}{|l|c|c|c|c|}
\hline \multicolumn{1}{|c|}{ ASPECTOS } & Género & Edad & Semestre & Turno \\
\hline Intensidad & $\mathbf{. 0 0 0}$ & .884 & $\mathbf{. 0 0 8}$ & $\mathbf{. 0 3 9}$ \\
\hline Frecuencia & $\mathbf{. 0 0 0}$ & .239 & $\mathbf{. 0 4 3}$ & .480 \\
\hline
\end{tabular}

Como se puede observar las variables género y semestre son las que establecen diferencias significativas en los dos aspectos indagados del estrés de examen, mientras que la variable edad no lo hace con ninguno.

Con base en los resultados obtenidos en el análisis de diferencia de grupo en el nivel de indicadores empíricos se puede establecer el siguiente perfil sociodemográfico y situacional del estrés de examen de los alumnos del Colegio de Bachilleres Lomas (en el caso de la variable edad la diferencia no fue posible establecerla entre los diferentes grupos):

Los alumnos del género femenino son los que consideran con mayor frecuencia como estresores la calificación que pudieran obtener, el tipo de preguntas, ejercicios, o problemas que se encuentran en el examen, la forma en que el profesor va a calificar el examen y olvidar lo que estudiaron en el momento del examen.

Los alumnos del género femenino son las que presentan con mayo frecuencia los siguientes síntomas: trastornos en el sueño (insomnio o pesadillas), fatiga crónica (cansancio permanente), problemas de digestión, dolor abdominal o diarrea, rascarse, morderse las uñas, frotarse, etc., somnolencia o mayor necesidad de dormir, sentimientos de depresión y tristeza (decaído), problemas de concentración y aumento o reducción del consumo de alimentos.

Los alumnos del género femenino son los que utilizan con mayor frecuencia las siguientes estrategias de afrontamiento: volver a repasar los apuntes o ejercicios para el examen, esforzarse por mantener la calma (respiración o concentración profunda), elogios a sí mismo (echarse porras y convencerse de que si puede), la religiosidad (realizar oraciones) y platicar con los compañeros (hablar sobre el examen, la materia, o el maestro).

Los alumnos del tercer y quinto semestre son los que presentan con mayor frecuencia el síntoma de somnolencia o mayor necesidad de dormir.

Los alumnos de quinto semestre son los que utilizan con mayor frecuencia las estrategias de volver a repasar los apuntes o ejercicios para el examen y esforzarse por 
mantener la calma (respiración o concentración profunda), mientras que los alumnos del tercer semestre son los que menos las utilizan.

Los alumnos de quinto semestre son los que utilizan con mayor frecuencia las estrategias de la religiosidad (realizar oraciones) y la de platicar con los compañeros (hablar sobre el examen, la materia o el maestro), mientras los que menos la utilizan son los del primer semestre.

Los alumnos del turno matutino son los que utilizan con mayor frecuencia las estrategias de esforzarse por mantener la calma (respiración o concentración profunda) y la religiosidad (realizar oraciones).

Con base en los resultados obtenidos en el análisis de diferencia de grupo en el nivel de componentes del estrés de examen se puede establecer el siguiente perfil sociodemográfico y situacional:

Los alumnos del género femenino son las que valoran con mayor frecuencia las demandas del entorno como estresores, presentan con mayor frecuencia los síntomas del estrés de examen y utilizan con mayor frecuencia las estrategias de afrontamiento.

Los alumnos de quinto semestre son los que utilizan con mayor frecuencia las estrategias de afrontamiento para enfrentar el estrés de examen.

Con base en los resultados obtenidos en el análisis de diferencia de grupo en el nivel del estrés de examen se puede establecer el siguiente perfil sociodemográfico y situacional:

Los alumnos del género femenino son los que presentan una mayor intensidad y frecuencia del estrés de examen.

Los alumnos de quinto semestre son los que presentan un mayor nivel de estrés de examen, mientras que los que lo presentan con un nivel menor son los alumnos de tercer semestre.

Los alumnos de quinto semestre son los que presentan con mayor frecuencia el estrés de examen, mientras que los que lo presentan con una frecuencia menor son los alumnos del primer semestre.

Los alumnos del turno matutino son los que presentan el estrés de examen con mayor intensidad.

En lo general, los resultados obtenidos en los tres niveles de análisis del estrés de examen, coinciden tendencialmente con los resultados obtenidos por Barraza y Acosta (2007), pero a diferencia de ese estudio, en el presente, la variable género y semestre impactan de manera más decisiva, al ser mayor el número de indicadores empíricos donde se manifiesta, lo que se refleja en los componentes y en los diferentes aspectos del estrés de examen. Coincidiendo con los citados autores las variables edad y turno son las que menos influencia tienen en el estrés de examen.

\section{Análisis correlacional}

El análisis correlacional se realizó con los tres niveles del estrés de examen, que se han venido manejando hasta este momento, y con la variable número de materias reprobadas. En todos los casos se consideró que un nivel de significación menor a .05 representaba que existía correlación con dicha variable.

a) Indicadores empíricos del estrés de examen

Los resultados obtenidos del análisis realizado en este nivel con la variable abordada se presentan en la siguiente tabla: 
Tabla No. 10 Resultados del análisis correlacional entre los indicadores empíricos del estrés de examen y el número de materias reprobadas

\begin{tabular}{|l|c|}
\hline \multicolumn{1}{|c|}{ INDICADORES } & Sig. \\
\hline El no haber estudiado previamente & $\mathbf{. 0 0 1}$ \\
\hline La calificación que pudieras obtener & .288 \\
\hline El tipo de preguntas, ejercicios, o problemas que se encuentran en el examen & .924 \\
\hline La falta de tiempo para terminar el examen & $\mathbf{. 0 3 1}$ \\
\hline La forma en que el profesor va a calificar el examen & .227 \\
\hline Olvidar lo que estudiaste en el momento del examen & .117 \\
\hline La vigilancia que hiciera el profesor para que no pudieras copiar & $\mathbf{. 0 0 0}$ \\
\hline Trastornos en el sueño (insomnio o pesadillas) & .642 \\
\hline Fatiga crónica (cansancio permanente) & .078 \\
\hline Problemas de digestión, dolor abdominal o diarrea & $\mathbf{. 0 0 8}$ \\
\hline Rascarse, morderse las uñas, frotarse, etc. & $\mathbf{. 0 0 1}$ \\
\hline Somnoliencia o mayor necesidad de dormir & .067 \\
\hline Sentimientos de depresión y tristeza (decaído) & $\mathbf{. 0 0 0}$ \\
\hline Problemas de concentración & $\mathbf{. 0 0 0}$ \\
\hline Sensación de tener la mente vacía (bloqueo mental) & $\mathbf{. 0 0 0}$ \\
\hline Sentimiento de agresividad o aumento de irritabilidad & $\mathbf{. 0 1 2}$ \\
\hline Conflictos o discusiones con mis compañeros & $\mathbf{. 0 0 8}$ \\
\hline Aislamiento de los demás & $\mathbf{. 0 1 2}$ \\
\hline Desgano para realizar las labores escolares & $\mathbf{. 0 0 0}$ \\
\hline Absentismo e impuntualidad en las clases & $\mathbf{. 0 0 0}$ \\
\hline Aumento o reducción del consumo de alimentos & $\mathbf{. 0 5 0}$ \\
\hline Volver a repasar los apuntes o ejercicios para el examen & $\mathbf{. 0 0 0}$ \\
\hline Esforzarse por mantener la calma (respiración o concentración profunda) & .716 \\
\hline Elogios a sí mismo (echarme porras y convencerme de que si puedo) & .557 \\
\hline Distracción evasiva (salir a caminar, comprar, saludar a alguien, etc.) & $\mathbf{. 0 2 3}$ \\
\hline La religiosidad (realizar oraciones) & .987 \\
\hline Platicar con los compañeros (hablar sobre el examen, la materia, o el maestro) & .051 \\
\hline
\end{tabular}

Como se puede observar la variable número de materias reprobadas se correlaciona positivamente con 16 de los 27 indicadores empíricos del estrés de examen lo que indica que el reprobar materias estresa con mayor frecuencia a los alumnos, manifestándose esto en más de la mitad de sus indicadores empíricos.

b) componentes del estrés de examen

Los resultados obtenidos del análisis realizado en este nivel con la variable abordada se presentan en la siguiente tabla:

Tabla No. 11 Resultados del análisis correlacional entre los componentes procesuales del estrés de examen y el número de materias reprobadas

\begin{tabular}{|l|c|}
\hline \multicolumn{1}{|c|}{ COMPONENTES } & $\begin{array}{c}\text { Número de materias } \\
\text { reprobadas }\end{array}$ \\
\hline Estresores & $\mathbf{. 0 0 2}$ \\
\hline Síntomas & $\mathbf{. 0 0 0}$ \\
\hline Estrategias de Afrontamiento & .279 \\
\hline
\end{tabular}

Como se puede observar la variable número de materias reprobadas se correlaciona positivamente con los componentes estresores y síntomas, lo cual coincide con lo reportado en el nivel de los indicadores empíricos.

c) Estrés de examen

Los resultados obtenidos del análisis realizado en los diferentes aspectos de este nivel con la variable abordada se presentan en la siguiente tabla: 
Tabla No. 12 Resultados del análisis correlacional entre los aspectos indagados del estrés de examen y el número de materias reprobadas

\begin{tabular}{|l|c|}
\hline \multicolumn{1}{|c|}{ ASPECTOS } & $\begin{array}{c}\text { Número de materias } \\
\text { reprobadas }\end{array}$ \\
\hline Intensidad & .963 \\
\hline Frecuencia & .000 \\
\hline
\end{tabular}

Como se puede observar la variable número de materias reprobadas únicamente se correlaciona positivamente con la frecuencia del estrés de examen, lo que se refleja sobre todo en los estresores y los síntomas y en 16 indicadores empíricos del estrés de examen.

Con relación al análisis correlacional realizado en los tres niveles del estrés de examen y el número de materias reprobadas se puede afirmar lo siguiente:

A mayor número de materias reprobadas con mayor frecuencia el alumno considera como estresores el no haber estudiado previamente, la falta de tiempo para terminar el examen y la vigilancia que hiciera el profesor para que no pudiera copiar.

A mayor número de materias reprobadas el alumno manifiesta con mayor frecuencia los siguientes síntomas: problemas de digestión, dolor abdominal o diarrea, rascarse, morderse las uñas, frotarse, etc., sentimientos de depresión y tristeza (decaído), problemas de concentración, sensación de tener la mente vacía (bloqueo mental), sentimiento de agresividad o aumento de irritabilidad, conflictos o discusiones con mis compañeros, aislamiento de los demás, desgano para realizar las labores escolares, absentismo e impuntualidad en las clases y aumento o reducción del consumo de alimentos.

A mayor número de materias reprobadas el alumno utiliza con mayor frecuencia las estrategias de volver a repasar los apuntes o ejercicios para el examen y distracción evasiva (salir a caminar, comprar, saludar a alguien, etc.).

A mayor número de materias reprobadas, los alumnos consideran con mayor frecuencia las demandas del entorno como estresores.

A mayor número de materias reprobadas los alumnos manifiestan con mayor frecuencia los síntomas del estrés de examen.

A mayor número de materias reprobadas el estrés de examen se presenta con mayor frecuencia.

\section{CONCLUSIONES}

Una vez finalizada la presente investigación se está en condiciones de concluir, con relación a cada objetivo, lo siguiente:

\section{Establecer el perfil descriptivo del estrés de examen de los alumnos de} educación media superior.

El 96.8\% de los alumnos del Colegio de Bachilleres Lomas algunas veces presentan el estrés de examen con una intensidad medianamente alta.

Las demandas del entorno que son valoradas con mayor frecuencia como estresores por los alumnos del Colegio de Bachilleres Lomas son la calificación que pudiera obtener y el tipo de preguntas, ejercicios, o problemas que se encuentran en el examen, Los síntomas que se presentan con mayor frecuencia entre los alumnos del Colegio de Bachilleres Lomas son problemas de concentración y rascarse, morderse las uñas, frotarse, etc. Las estrategias de afrontamiento que son utilizadas con mayor 
frecuencia por los alumnos del Colegio de Bachilleres Lomas son platicar con los compañeros (hablar sobre el examen, la materia, o el maestro) y volver a repasar los apuntes o ejercicios para el examen,

Identificar las materias que más estrés provocan en los alumnos de educación media superior al momento de presentar un examen.

Más del 50\% de los alumnos reportan presentar estrés de examen con la materia de matemáticas (o relacionada con las matemáticas).

Distinguir las variables sociodemográficas y situacionales que establecen diferencias significativas en el estrés de examen de los alumnos de educación media superior.

La variable género establece diferencias significativas en 17 de 27 indicadores empíricos del estrés de examen, en sus tres componentes procesuales y en la intensidad y frecuencia del estrés de examen.

La variable turno únicamente establece diferencias significativas en dos indicadores empíricos del estrés de examen.

La variable semestre establece diferencias significativas en las estrategias de afrontamiento y en la intensidad y frecuencia del estrés de examen.

Determinar la relación que existe entre el estrés de examen y el número de materias reprobadas en los alumnos de educación media superior.

La variable número de materias reprobadas se correlaciona positivamente con 16 de los 27 indicadores empíricos del estrés de examen, con los componentes estresores y síntomas y con la frecuencia con que se presenta el estrés de examen.

Estos resultados se integran al trabajo que, de manera incipiente, venimos desarrollando en el campo del estrés de examen, pero que creemos sirve de muestra, y de aliciente, para futuros trabajos, encuadrados éstos en las líneas de investigación que hemos derivado para el estrés académico y el estrés de examen a partir de la construcción del modelo sistémico cognoscitivista.

\section{REFERENCIAS}

Barraza Macías Arturo (2005), Características del estrés académico en alumnos de educación media superior, Revista Electrónica Psicologíacientífica.Com

Barraza Macías Arturo (2006), Un modelo conceptual para el estudio del estrés académico, Revista Electrónica de Psicología Iztacala, Vol. 9. No. 3, pp. 110129

Barraza Macías Arturo y Acosta Chávez Magdalena (2007), El estrés de examen en educación media superior. Caso Colegio de Ciencias y Humanidades de la Universidad Juárez del Estado de Durango, Innovación Educativa, Vol. 7, No. 37.

Barraza Macías Arturo (2007a), Estrés académico. Un estado de la cuestión, Revista Electrónica Psicologíacientífifca.Com

Barraza Macías Arturo (2007b), El campo de estudio del estrés. Del Programa de Investigación Estímulo-Respuesta al Programa de Investigación PersonaEntorno, Revista Internacional de Psicología, Vol. 8 No. 2.

Benjamin Stora Jean (1992), El estrés, México, Presses Universitaires de France y Publicaciones Cruz.

Campos Vargas Jasón David, Ocampo Solís José Alberto, Padilla Vindas Juan José, Corrales Araya María Antonieta y Colindres Molina Dilia (2005), Estrategias del 


\section{(c) (i)}

Este texto está protegido por una licencia CreativeCommons

Usted es libre para Compartir - copiar y redistribuir el material en cualquier medio o formato - y Adaptar el documen- to - remezclar, transformar y crear a partir del material- para cualquier propósito, incluso comercialmente, siempre que cumpla la condición de:

Atribución: Usted debe reconocer el crédito de una obra de manera adecuada, proporcionar un enlace a la licencia, e in- dicar si se han realizado cambios. Puede hacerlo en cualquier forma razonable, pero no de forma tal que sugiera que tie- ne el apoyo del licenciante o lo recibe por el uso que hace. 
afrontamiento del estrés y rendimiento académico en adolescentes, Boletín Salud Integral y Movimiento Humano, No. 10.

Cohen S. y Lazarus R. S. (1979) "Coping with the stresses of illness", en Stone G. C., Cohen S. y Adler N. E. (Eds), Health Psychology: a handbook San Francisco: Jossey-Bass.

Elliot, G. R., y Eisdorfer, C. (1982). Stress and human health. New Cork, USA, Springer Verlag.

Hernández Sampìeri Roberto, Fernández Collado Carlos y Baptista Lucio Pilar (1998), Metodología de la Investigación, México, Mc Graw Hill.

Hogan Thomas P. (2004), Pruebas psicológicas, México, El Manual Moderno.

Karasek Robert (1979), Job demands, job decision latitude and mental strain implications for job redesign. Administrative Science Quaraterly, No. 24, pp. 285-306.

Karasek Robert (2001), El modelo de demandas/control: enfoque social, emocional y fisiológico del riesgo de estrés y desarrollo de comportamientos activos, Enciclopedia de Salud y Seguridad en el Trabajo, Vol. 2, No. 34, pp. 6-15.

Lazarus, R. S., y S. Folkman (1986), Estrés y procesos cognitivos. Barcelona, España, Martínez Roca.

Lazarus, Richard S. (2000), Estrés y emoción. Manejo e implicaciones en nuestra salud, Bilbao, España, Biblioteca de Psicología - Editorial Desclée De Brouwer.

Namakforoosh Mohammad Naghi (2002), Metodología de la investigación, México, Limusa.

Pellicer Olga; Salvador Alicia y Benet Isabel A. (2002), Efectos de un estresor académico sobre las respuestas psicológica e inmune en jóvenes, Psicothema Vol. 14, No. 2, pp. 317-322

Salgero Gómez Alicia (1989), Incidencia del estrés y la ansiedad en una muestra de estudiantes universitarios de ciencias y letras, Base de Datos REDINED (on line) (recuperado en marzo de 2005)

Theorell T. y Karasek R. (1996), Currents issues relating to psychosocial job strain and cardiovascular disease research, Journal of Occupational Health Psychology, Vol. 1, No. 1, pp. 9-26

Travers Cheryl J. y Cary L. Cooper (1997), El estrés de los profesores, Barcelona, España, Paidós.

Viñas Poch F. y Caparrós Caparrós B. (2000), Afrontamiento del período de exámenes y sintomatología somática autoinformada en un grupo de estudiantes universitarios, Revista Psicología.com, Vol. 4, No. 1. 